\title{
Test stimulus sequence effects on object orientation and line tilt generalization
}

\author{
PATRICK A. CABE \\ University of North Carolina, Chapel Hill, North Carolina 27514
}

\begin{abstract}
Four pigeons, trained to discriminate the presence of an object in a tunnel vs. the object's absence, were tested for generalization over orientations around the vertical axis of the object where the test orientations were presented in randomized blocks or in an ordered sequence. Gradients showed sharpening over repeated generalization test sessions, but only with the ordered test sequence. Final gradients were strongly peaked at the training orientation and were strongly asymmetrical. Gradients obtained with randomized sequences remained flat through all tests. Four other birds trained and tested in a parallel fashion with a line tilt continuum showed some sharpening across repeated generalization tests, although gradients obtained were clearly peaked in the first test session. No differences between random and ordered test stimulus sequences were seen. Both kinds of sequences produced clearly symmetrical gradients, with peaks at the training orientation of the line.
\end{abstract}

A persistent suggestion in the literature of stimulus generalization is that the process has adaptive value in natural environments. The stimulus conditions that might affect the behavior of organisms change across time, and the ability of an organism to respond to stimulus situations similar to those it has encountered in the past presumably promotes that organism's survival.

Changes in a natural environment, however, differ from those to which an organism is exposed in typical laboratory tests of stimulus generalization in two important ways: changing stimuli in a natural environment are best characterized as discrete objects that undergo transformations of various sorts, while stimuli in laboratory tests of stimulus generalization are typically derived from relatively abstract continua (e.g., wavelength of light). Moreover, changes in a natural environment are quite likely to be continuous or ordered in time, while stimuli in laboratory tests are generally randomly sequenced.

One might ask, then, whether generalization gradients over transformations of discrete objects differ from gradients obtained using more conventional stimulus continua and whether ordered sequences of generalization test stimuli produce gradients that differ from those produced by random sequences.

The two studies reported here tested stimulus generalization over rotation around a vertical axis of a three-dimensional free-standing object (Experiment 1) and over line tilt orientation in the frontal-parallel plane (Experiment 2), under random vs. ordered test stimulus sequences.

This work was carried out while the author held a National Institute of Mental Health postdoctoral fellowship. Reprints may be obtained from the author at the National Institute of Environmental Health Sciences, P.O. Box 12233, Research Triangle Park, North Carolina 27709.

\section{EXPERIMENT 1: GENERALIZATION OVER OBJECT ORIENTATION}

\section{Method}

Subjects. Four nonnaive adult male white Carneaux pigeons were used. Experimental histories did not include any previous visual discrimination or stimulus generalization experience. All birds were maintained at approximately $75 \%$ of free-feeding weight throughout the experiment, with ad-lib water and health grit in the home cage. Supplemental food was provided after each session to maintain the $75 \%$ weight.

Apparatus. The experiment was conducted using a modified picnic hamper operant conditioning chamber, fitted with a single $2.5-\mathrm{cm}$ clear glass response key (Cabe, 1976) centered about $20 \mathrm{~cm}$ above the floor. The key operated as a normally closed switch requiring about $30 \mathrm{~g}$ of pressure to open. A food magazine to provide mixed grain reinforcers, lighted during food presentations, was centered about $10 \mathrm{~cm}$ below the key. A low level of white masking noise was provided.

A short tunnel was mounted against the back side of the response panel. Birds viewed the interior of the tunnel through the clear glass key. The stimulus object used could be rotated into and out of the end of the tunnel by means of a small reversible electric motor and a pulley and belt arrangement. The object was attached to a T-shaped panel rotating around the intersection of the $T$. Thus, two equal-sized right-angle alternative "ends" of the tunnel could be presented. The stimulus object, a small free-standing wooden block $(5.7 \mathrm{~cm}$ tall $\times 1.9 \times 1.9 \mathrm{~cm}$, with a $1.9-\mathrm{cm}$ hemicylindrical projection centered on one vertical face), modeled after Lumsden and Pullen (1970), was mounted on one side of the $T$ panel. Rotation of the panel allowed an object-present/object-absent $\left(S^{D} / S^{\Delta}\right)$ discrimination.

The object was mounted with the projection oriented $45 \mathrm{deg}$ to the subject's left. Orientation of the object in generalization testing was varied by mounting the object on the indexing mechanism of a 12-pole rotary switch, which was operated by hand in the generalization test sessions. The generalization stimuli consisted of the training orientation plus five others in $60-$ deg steps from the training orientation.

Timing, recording, and logical functions were implemented using standard electromechanical components located in another room. 
Procedure. Since the birds had all been run in similar chambers before, no pretraining was necessary. All birds received 7 consecutive days of discrimination training on the $S^{D} / S^{\Delta}$ problem, in a manner analogous to that employed by Jenkins and Harrison (1960). Each daily session consisted of 72 30-sec trials, with $S^{D}$ and $S^{\Delta}$ alternating in a quasirandom sequence such that neither display appeared more than three times in succession (Fellows, 1967). A 10-sec intertrial interval (ITI) intervened between trials, during which the $T$ panel described above was rotated. Reinforcement (5-sec access to grain) was available on a variable-interval (VI) 1 -min schedule during $S^{D}$ trials; no reinforcement was available during $S^{\Delta}$ trials. Reinforcers not collected at the end of a trial were cancelled. Reinforcer presentations were not added to the trial length, but if the food magazine was operating at the end of a trial, the succeeding ITI was postponed until the end of the reinforcer cycle. Counts of responses in $S^{D}$ and $S^{\Delta}$ trials were collected by means of a printout counter.

Generalization testing began on Day 8 . All birds received three initial trials in which one reinforcer per trial was given in the presence of the $\mathrm{S}^{\mathrm{D}}$ display. Birds tested with random sequences then proceeded through 72 presentations of the generalization displays in randomized blocks of six orientations of the object. Birds in the ordered sequence condition also received 72 generalization presentations, beginning with the $\mathbf{S}^{\mathbf{D}}$ display and progressing through the remaining displays in an ordered stepwise fashion, the rotation of the object always being from the subject's left to right.

Generalization test sessions were repeated at 3-day intervals; 2 days of retraining on the initial discrimination intervened between generalization test sessions. All generalization tests were carried out with no food available after the initial three warm-up trials. Responses to each of the orientations were summed across all trials in each test session. A total of five generalization tests were conducted, and generalization gradients were constructed for each bird in each test session.

\section{Results}

All birds showed clear evidence of having learned the initial discrimination. Percentages of total responses to the $\mathrm{S}^{\mathrm{D}}$ display ranged from $76 \%$ to $89 \%$ (mean $82 \%$ ) on the last day of the initial discrimination training. Retraining between generalization test sessions brought discrimination back within this range in all cases.

Figure 1 shows the relative gradients produced in the five generalization test sessions under the random and ordered sequencing procedures: The remarkable result to be seen here is that there is a striking difference in the shapes of the gradients obtained under the two sequence conditions. It is also clear that the ordered presentations ultimately give very sharp but asymmetrical gradients, and that the gradients do not differ between animals.

\section{EXPERIMENT 2: GENERALIZATION OVER LINE TILT}

\section{Method}

Subjects. Four other birds with about the same histories as those in the previous experiment were used. Again, no bird's history included discrimination or stimulus generalization experience.

Apparatus. A second single-key operant conditioning chamber was used, set up in the same way as in the previous experiment with the exception that the stimulus displays used were $.3-\mathrm{cm}$ white stripes across a $2.5-\mathrm{cm}$ black field, rearprojected by means of a standard IEEE in-line projector on to the translucent key.

The $S^{D}$ display was a line at $45-$ deg clockwise from vertical. The $S^{\Delta}$ display was a blank white key. The generalization test displays consisted of the $S^{D}$ display plus seven similar lines varying from the $S^{D}$ orientation in 45-deg steps.

Timing, recording, and logic control functions were by means of electromechanical equipment located in another room.
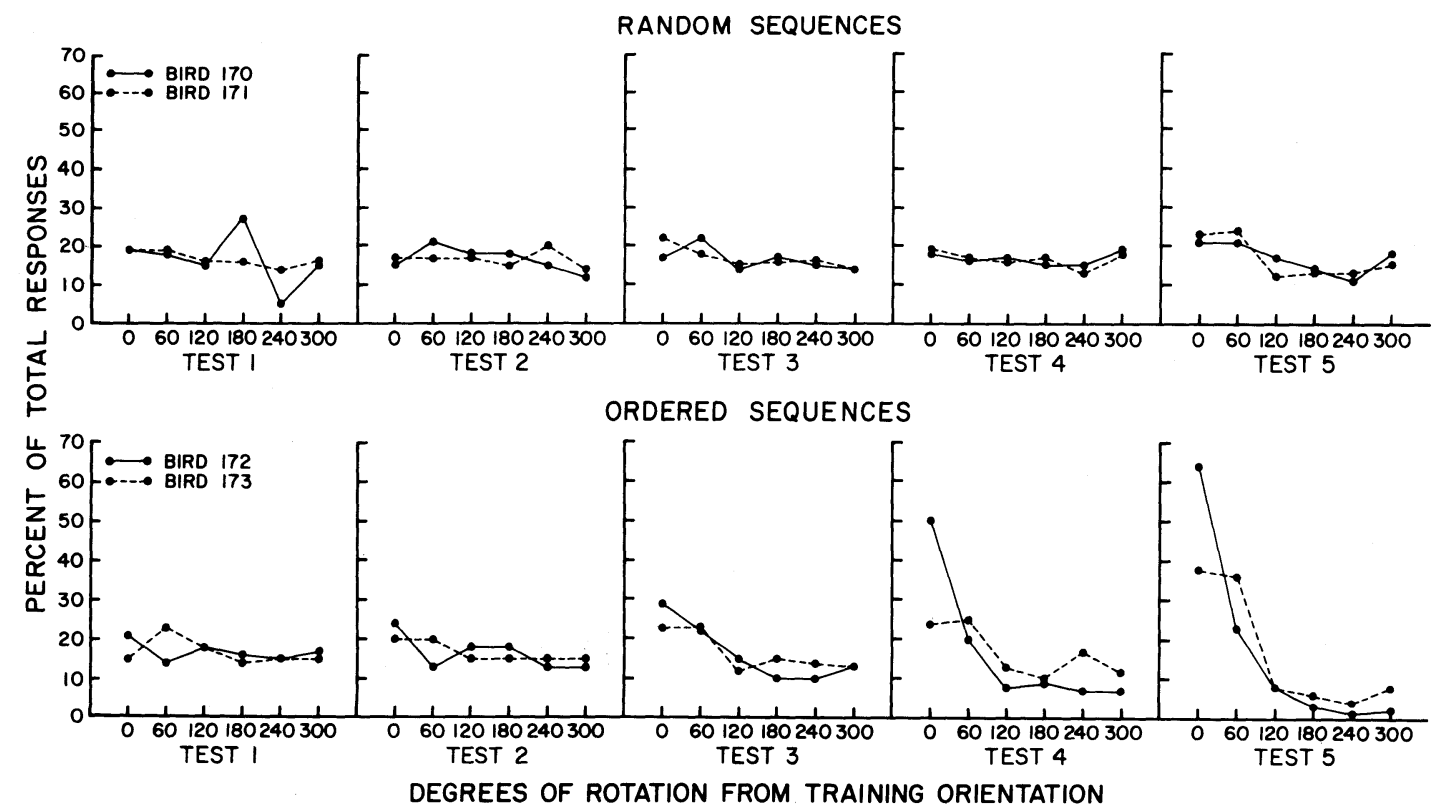

Figure 1. Relative generalization gradients for individual birds obtained with random vs. ordered generalization test stimuli for the object orientation dimension. 
Procedure. Initial discrimination training paralleled that given in the previous experiment; in both cases the procedure was analogous to that used by Jenkins and Harrison (1960). Again, each of seven daily sessions consisted of 7230 -sec stimulus presentations, $S^{D}$ and $S^{\Delta}$ alternating in a quasirandom fashion. A 10-sec ITI again intervened between trials. Responses in each trial were recorded using a printout counter.

A VI 1-min schedule of reinforcment was in effect during $S^{D}$; responses in $S^{\Delta}$ were never reinforced. Reinforcers were not collected at the end of a trial were cancelled, and reinforcer presentations did not increase the length of a trial, although a feeder operation at the end of a trial postponed the ITI.

Generalization testing began on Day 8. Generalization testing also paralleled that used in the previous experiment except that there were eight generalization test displays. Again, three reinforced presentations of $S^{\mathbf{D}}$ began each generalization test session. Birds in the random sequence condition then received 72 presentations of the generalization test displays, in blocks of 8 . Birds in the ordered sequence received 72 ordered presentations of the eight test displays, beginning with the $\mathrm{S}^{\mathrm{D}}$ display and progressing stepwise through the series. Two days of retraining intervened between generalization tests. Two birds received random sequences of test presentations and two birds ordered sequences.

\section{Results}

All birds showed near-perfect discrimination on the last initial discrimination training session, with percentages of total responses to $\mathrm{S}^{\mathrm{D}}$ ranging upward from $99 \%$.

Figure 2 shows the results of three generalization tests for the line tile experiment; this series was termi-
Table 1

Total Responses Emitted by Individual Birds in Generalization

\begin{tabular}{|c|c|c|c|c|c|c|}
\hline \multirow[b]{2}{*}{ Bird } & \multirow{2}{*}{$\begin{array}{l}\text { Sequence } \\
\text { Condition }\end{array}$} & \multicolumn{5}{|c|}{ Generalization Test } \\
\hline & & 1 & 2 & 3 & 4 & 5 \\
\hline \multicolumn{7}{|c|}{ Object Orientation (Experiment 1 ) } \\
\hline $\begin{array}{l}170 \\
171 \\
172 \\
173\end{array}$ & $\begin{array}{l}\text { Random } \\
\text { Random } \\
\text { Ordered } \\
\text { Ordered }\end{array}$ & $\begin{array}{r}158 \\
1208 \\
613 \\
873\end{array}$ & $\begin{array}{r}508 \\
1977 \\
574 \\
1417\end{array}$ & $\begin{array}{r}1052 \\
1319 \\
887 \\
1039\end{array}$ & $\begin{array}{r}1262 \\
1668 \\
634 \\
1261\end{array}$ & $\begin{array}{r}1000 \\
1705 \\
439 \\
766\end{array}$ \\
\hline \multicolumn{7}{|c|}{ Line Tilt (Experiment 2) } \\
\hline $\begin{array}{r}87 \\
143 \\
144 \\
145\end{array}$ & $\begin{array}{l}\text { Random } \\
\text { Random } \\
\text { Ordered } \\
\text { Ordered }\end{array}$ & $\begin{array}{c}339 \\
216 \\
37 * \\
209\end{array}$ & $\begin{array}{l}775 \\
529 \\
601 \\
691\end{array}$ & $\begin{array}{l}790 \\
599 \\
470 \\
295\end{array}$ & & \\
\hline
\end{tabular}

*Not plotted in Figure 1.

nated when the gradients appeared to be asymptotic. Two things can be noted. First, for both the ordered and the random sequences, the gradients are nicely peaked at the $S^{D}$ orientation and are nicely symmetrical. Second, there is no difference in the shapes of the two sets of gradients. Again, the gradients are similar for all birds.

\section{DISCUSSION}

The results of these two experiments show that ordered sequences of generalization test stimuli produce strongly peaked

\section{RANDOM SEQUENCES}

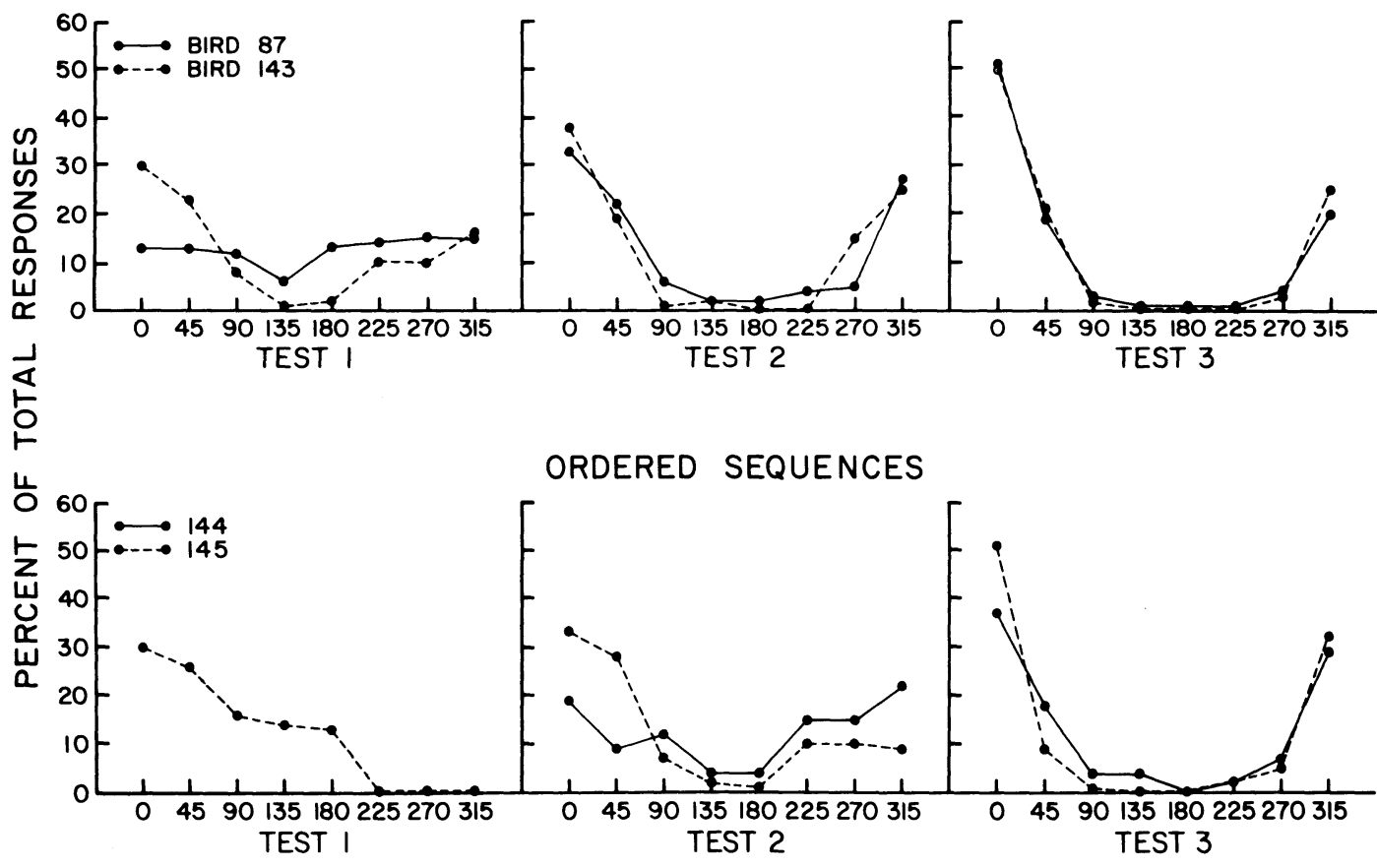

DEGREES OF LINE TILT FROM TRAINING ORIENTATION

Figure 2. Relative generalization for individual birds obtained with random vs. ordered generalization test stimuli for the line tilt dimension. Bird 144 emitted too few responses to plot in the first generalization test. 
and markedly asymmetrical generalization gradients when the continuum is object orientation, but that ordered and random sequences produce equivalent gradients when the continuum is line tilt. The line tilt result is in accord with the only other apparent test of generalization using ordered sequences of test stimuli, a study by Stevenson (1966) on wavelength generalization.

The results of these two experiments suggest at least a methodological point. The typical procedure of randomizing presentations in generalization testing does not appear to be critical to the development of generalization gradients. In fact, the present results imply that ordered presentations in generalization are as good as, and under some conditions perhaps better than, random sequences.

Herrnstein, Loveland, and Cable (1976), in discussing the ability of pigeons to categorize disparate instances of complex concepts, make a point that may apply with regard to the results presented here. Herrnstein et al. (1976) point out that it has been assumed that "the process of discrimination is separable from the things being discriminated, even though there is reason to examine the possibility that, at least sometimes, the two are inseparably linked" (p. 286). Insofar as discrimination and generalization are inverse processes, a parallel statement for stimulus generalization can be made. The difference in gradients obtained with solid object rotation and line tilt continua provide a possible example of a linkage between process and continuum in stimulus generalization. Herrnstein et al. suggest that pigeons are evolutionarily organized to differentiate certain classes of objects. The present work tentatively suggests that pigeons may be evolutionarily organized to be sensitive to certain classes of transformations.

An interesting problem related to explanations of stimulus generalization gradients arises from the object orientation results. Lashley and Wade (1946) proposed that generalization gradients depend on comparisons among the test stimuli. Later investigators have attempted to falsify this position (e.g., Thomas \& Konick, 1966) by showing that peaked gradients do or do not result depending on opportunity for comparison among stimuli. The present results speak to the same issue in a different way: In the object orientation experiment, all birds received equal exposure to all object orientations, but the ordered sequence provided (at least in principle) less opportunity for comparison of orientations, since some orientations were always "further away" in time than others. One might expect, therefore, that a less steep gradient would result with ordered sequences than with random sequences, because in the random sequence any orientation could precede or follow any other about equally, presumably maximizing opportunity for comparison. Further, since in the ordered sequence the 60-deg orientation and the $300-\mathrm{deg}$ orientation were equally distant (in time) from the 0 -deg orientation $\left(S^{D}\right)$ and, consequently, presumably equally comparable to the 0 -deg orientation, the gradient should have been symmetrical around the o-deg orientation, which it clearly was not. The implication is that is that comparison processes are not prerequisite to stimulus generalization gradients of nonzero slope.

\section{REFERENCES}

CABE, P. A. An inexpensive glass response key for use with pigeons. Behavior Research Methods \& Instrumentation, 1976, $8,341$.

Fellows, B. J. Chance stimulus sequences for discrimination tasks. Psychological Bulletin, 1967, 67, 87-92.

Herrnste in, R. J., Loveland, D. H., \& CABLe, C. Natural concepts in pigeons. Journal of Experimental Psychology: Animal Behavior Processes, 1976, 2, 285-302.

Jenkins, H. M., \& Harrison, R. H. Effect of discrimination training on auditory generalization. Journal of Experimental Psychology, 1960, 59, 246-253.

LAshley, K. S., \& WADE, M. The Pavlovian theory of generalization. Psychological Review, 1946, 53, 72-87.

Lumsden, E. A., \& Pullen, M. R. Object orientation as a dimension of stimulus generalization. Psychonomic Science, $1970,19,55-56$.

Stevenson, J. G. Stimulus generalization: The ordering and spacing of test stimuli. Journal of the Experimental Analysis of Behavior, 1966, 9, 457-468.

Thomas, D. R., \& Konick, D. S. A comparison of different measures of response strength in the study of stimulus generalization. Journal of the Experimental Analysis of Behavior, $1966,9,239-242$.

(Received for publication March 26, 1979.) 